\title{
Modeling the crystallization kinetics of polymers displaying high levels of secondary crystallization
}

\author{
Catherine A. Kelly (i) $^{1} \cdot$ Mike J. Jenkins ${ }^{1}$ \\ Received: 10 June 2021 / Revised: 4 October 2021 / Accepted: 10 October 2021 / Published online: 19 November 2021 \\ (c) The Author(s) 2021. This article is published with open access
}

\begin{abstract}
The isothermal crystallization of poly(3-hydroxybutyrate-co-3-hydroxyvalerate) was evaluated using a range of models, namely, Avrami, simplified Hillier, Tobin, Malkin, Urbanovici-Segal, Velisaris-Seferis, and Hay. Two methods of model evaluation were used: determination of the parameters through traditional double log plots and curve fitting via nonlinear, multivariable regression. Visual inspection of the cumulative crystallization curves, calculation of the $\mathrm{R}^{2}$ value and standard error of the regression, and evaluation of the returned parameters were used to assess which model best describes the experimental data. The Hay model was found to generate the best fit, closely followed by the Velisaris-Seferis parallel model, suggesting that primary and secondary crystallization occur concurrently. The Avrami, Malkin, and Tobin models were found to perform well when the data is restricted to the region where primary crystallization dominates; however, they could not be used to successfully model the entire crystallization process. This work highlights the importance of selecting the most appropriate model for analyzing kinetics, especially when high levels of lamellar thickening and infilling occur during crystallization.
\end{abstract}

\section{Introduction}

The kinetics of crystallization are an important consideration in polymer processing because they significantly affect the final thermal and mechanical properties of a material. If primary crystallization occurs slowly, when cooling from the melt phase, only a few nuclei are generated that grow into large spherulites, rendering the material brittle. As the rate of crystallization is dependent on the degree of undercooling from the melt, knowledge of the kinetics allows precise control of material morphology during processing.

Polymer crystallization consists of two processes: primary and secondary. Primary crystallization is initiated in the amorphous polymer through the formation of nuclei from small regions of chain entanglements. Growth of crystalline

Supplementary information The online version contains supplementary material available at https://doi.org/10.1038/s41428021-00581-0.

Catherine A. Kelly

c.a.kelly@bham.ac.uk

1 School of Metallurgy and Materials, University of Birmingham, Edgbaston, Birmingham B15 2TT, UK lamella proceeds from these nuclei to form spherulites that expand radially until they begin to impinge onto one another, marking the end of the primary process. The secondary crystallization process relates to thickening of the lamella due to reorganization of the crystal structure and the formation of small crystalline structures within the amorphous regions, known as infilling. This secondary process has been reported to follow a logarithmic time scale [1]; more recently, a square root time dependency has been found, consistent with a diffusion-controlled process [2].

The Avrami model is commonly used to determine the crystallization kinetics of polymers, where the Avrami exponent $\left(n_{a}\right)$ provides information for the nucleation and growth mechanisms [3-5]. Limitations have been found to this model, including only being applicable to primary crystallization and meaningless fractional $\mathrm{n}_{\mathrm{a}}$ values [6-8]. Alternatives, namely, the simplified Hillier [9, 10], Tobin [11-13], Malkin [14], and Urbanovici-Segal [15, 16] models, many of which are extensions or adaptations of the standard Avrami analysis, have been proposed with the aim of accounting for the presence of secondary crystallization. Supaphol has previously analyzed the effectiveness of these models for the isothermal crystallization of syndiotactic polypropylenes $[8,17]$ with others comparing combinations of these models for pure polymers [10, 14, 16, 18-20], 
blends [21-23] and nanocomposites [24-26]. Following an in-depth review of these papers, no singular model has been found to be consistently the best over a wide range of polymers. In addition, none of these analyses have been performed for polymers with substantial secondary crystallization, and where such crystallization is present, the data is limited to considering the primary crystallization region in isolation [25].

Kelly et al. have previously shown the Avrami model to be unsuitable for poly(3-hydroxybutyrate-co-3-hydroxyvalerate) (PHB-co-HV) as a result of the high degree of secondary crystallization that follows the primary mechanism [27]. The researchers proposed an alternative method, the Hay model, giving meaningful integer $n_{a}$ and $R^{2}$ values in excess of 0.995 for PHB-co-HV. In addition, Velisaris and Seferis developed a model specifically to account for the primary and secondary crystallization processes occurring in either parallel or series [28]. This theory has been shown to be more effective than both the Avrami and Malkin models in the modeling of the isothermal crystallization of polypropylene and polypropylene composites containing talc [29].

Poly(3-hydroxybutyrate-co-3-hydroxyvalerate) (PHBco-HV) is a bacterially synthesized polymer that is formed via an energy storage mechanism when specific bacteria are fed a sugar source in the absence of key nutrients [30,31]. This sustainable production method, coupled with nontoxic, biocompatible, and biodegradable properties, has led to possible applications in the packaging industry [32]. However, a relatively low glass transition temperature of approximately $4{ }^{\circ} \mathrm{C}$ results in secondary crystallization at room temperature, resulting in progressive embrittlement and hindering widespread use [33, 34]. A thorough understanding of all aspects of the crystallization process for PHB-co-HV is paramount to be able to create suitable packaging materials; therefore, it is important to ensure that the most suitable models are utilized.

In this paper, the isothermal crystallization of PHB-co$\mathrm{HV}$ is analyzed using the Avrami, simplified Hillier, Tobin, Malkin, Urbanovici-Segal, Velisaris-Seferis, and Hay models. Nonlinear regression is utilized to determine the fit of the models, and the significance of the parameters obtained and the suitability of each model are discussed. Finally, the most suitable model for the analysis of PHB-co$\mathrm{HV}$ is proposed.

\section{Theoretical background}

Differential scanning calorimetry (DSC) is often used to study isothermal crystallization. As the evolution of crystallinity is directly proportional to the heat released during the process, the relative crystallinity as a function of time, $\theta(t)$ can be obtained through integration of the crystallization exotherms [17]. It is important, especially in cases of prolonged isothermal crystallization experiments with heat-labile polymers, that the temperatures selected do not lead to degradation of the material during the analysis. Many models have been proposed that can be used to analyze the development of crystallinity during primary and secondary processes.

The Avrami equation (Eq. (1)) is the most widely used equation to analyze the crystallization kinetics of polymers; however, it is only suitable for the primary mechanism, resulting in noninteger values being regularly determined for the Avrami exponent [8, 14]. The parameters of the Avrami equation can be determined from the gradient $\left(n_{a}\right)$ and antilogarithm of the y-intercept $\left(k_{a}\right)$ of a double log plot of $\log \left(-\ln \left(1-X_{t}\right)\right)$ versus log time.

$X_{t}=\left(1-e^{-k_{a} t^{n_{a}}}\right)$

where $X_{t}$ is the fractional crystallinity at time $t ; k_{a}$ is the Avrami rate constant; and $n_{a}$, which should be an integer between one and four, is the Avrami exponent representing the mechanism of nucleation and geometry of growth.

The simplified Hillier model (Eq. (2)) aims to limit the data to the primary process alone $[9,10]$. This is achieved by incorporating a value, $X_{p, \infty}$ for the degree of crystallinity at the conclusion of the primary process. The value of $X_{p, \infty}$ is taken from the intersection of tangents drawn to the primary and secondary regions of fractional crystallinity versus time plots [35]. The parameters of the Hillier equation can be determined from the gradient $\left(n_{a}\right)$ and antilogarithm of the y-intercept $\left(k_{a}\right)$ of a double log plot of log $\left(-\ln \left(1-X_{t} / X_{p, \infty}\right)\right)$ versus log time.

$X_{t}=X_{p, \infty}\left(1-e^{-k_{a} t^{n_{a}}}\right)$

where $X_{t}$ is the fractional crystallinity at time $t ; X_{p, \infty}$ is the fractional crystallinity at the end of the primary process; $k_{a}$ is the Avrami rate constant; and $n_{a}$ is the Avrami exponent.

Tobin modified the Avrami equation in an attempt to improve the modeling for the latter stages of crystallization by considering impingement of the spherulites. The resulting equation (Eq. (3)) also contains both a rate constant $\left(k_{t}\right)$ and exponent $\left(n_{t}\right)$, which have similar physical meanings to the Avrami model with the exception that $n_{t}$ does not need to be an integer [11-13]. The parameters of the Tobin equation can be determined from the gradient $\left(n_{t}\right)$ and antilogarithm of the y-intercept $\left(k_{t}\right)$ in a double log plot of $\log \left(X_{t} /\left(1-X_{t}\right)\right)$ versus log time.

$X_{t}=\frac{k_{t} t^{n_{t}}}{1+\left(k_{t} t^{n_{t}}\right)}$

where $X_{t}$ is the fractional crystallinity at time $t ; k_{t}$ is the Tobin rate constant; and $n_{t}$ is the Tobin exponent. 
Malkin derived his model (Eq. (4)) based on the dependence of the overall rate of crystallization on the sum of the rates of primary nuclei generation and crystal growth [14]:

$X_{t}=1-\frac{C_{0}+1}{C_{0}+e^{C_{1} t}}$

where $C_{0}$ is the Malkin exponent and is proportional to the ratio of the primary crystal growth rate to the primary nucleation rate and $C_{1}$ is the Malkin crystallization rate constant, and is related to the addition of various fractions of these two parameters [20].

Unlike previous models, these parameters can only be determined through curve fitting procedures; however, they can be estimated from the Avrami parameters, as illustrated in Eqs. (5) and (6) [8]:

$$
C_{0}=4^{n_{a}}-4
$$

$C_{1}=\ln \left(4^{n_{a}}-2\right)\left(\frac{k_{a}}{\ln (2)}\right)^{1 / n_{a}}$

Urbanovici and Segal [15] derived a model (Eq. (7)) to describe the crystallization process, which simplifies to the general Avrami equation when $r$ tends to one. The parameters of this model can only be determined via curve fitting.

$X_{t}=1-\left[1+(r-1)\left(k_{u s} t\right)^{n_{u s}}\right]^{1 /(1-r)}$

where $\mathrm{k}_{u s}$ and $n_{u s}$ are the Urbanovici-Segal rate constant and the Urbanovici-Segal exponent, respectively, and $r$ is a parameter that satisfies the condition $r>0$.

In addition to these equations, researchers have also devised models to specifically account for the secondary crystallization process. The Velisaris-Seferis model is based on two distinct regions observed in the Avrami double log plot, which are attributed to primary and secondary crystallization [28]. It describes how these processes can occur in either parallel or series, giving rise to two separate equations (Equations (8) and (9), respectively) based on the standard Avrami model and apportioned to the fraction of each process that has occurred at time $t$. The parameters of these equations can only be determined via curve fitting.

Parallel : $\frac{X_{t}}{X_{\infty}}=w_{p}\left(1-e^{-k_{p} t^{n_{s s}}}\right)+w_{s}\left(1-e^{-k_{s} t^{m_{i s}}}\right)$

Series : $\frac{X_{\infty}}{X_{t}}=\frac{w_{p}}{1-e^{-k_{p} t^{n_{s s}}}}+\frac{w_{s}}{1-e^{-k_{s} t_{t_{v s}}}}$

where $X_{t}$ and $X_{\infty}$ are the fractional crystallinities at time $t$ and infinite time, respectively; $w_{p}$ and $w_{s}$ are the weight factors for the primary and secondary processes, respectively, where the sum of these two factors equals $1 ; k_{p}$ and $k_{s}$ are the rate constants of the primary and secondary processes, respectively; and $n_{v s}$ and $m_{v s}$ are the exponents for both processes.

Recently, a new model, the Hay model, has been proposed that assumes that both processes occur concurrently and that the total crystallinity at time $t$ is the sum of the two individual contributions. The primary crystallization mechanism follows the standard Avrami equation, whereas the secondary crystallization is based on a square root dependence of fractional crystallinity, as reported previously [2]. This has led to the following Hay model (Eq. (10)) [36, 37] and, more recently, a new method to enable this theory to be applied to isothermal studies performed by DSC [27]. The parameters in this equation can be determined through a series of steps involving separation of the primary and secondary crystallization processes and evaluation of the primary data by the Avrami model in isolation [27].

$X_{t}=X_{p, \infty}\left(1-e^{-k_{p} t^{n a}}\right)\left(1+k_{s} t^{1 / 2}\right)$

where $X_{t}$ and $X_{p, \infty}$ are the fractional crystallinities at time $t$ and at the end of the primary process, respectively; $k_{p}$ and $k_{s}$ are the Avrami rate constants of the primary and secondary processes, respectively; and $n_{a}$ is the Avrami exponent.

In this paper, each of the models discussed is applied to PHB-co-HV, which is known to display significant secondary crystallization. The possible parameters are determined through traditional double log plots or calculated from the Avrami parameters, and curve fitting procedures are applied. A range of methods are applied to compare the models and determine which are best suited to polymers displaying significant degrees of secondary crystallization.

\section{Materials and experimental methods}

\section{Materials}

Poly(3-hydroxybutyrate-co-3-hydroxyvalerate) pellets containing 3\% 3-hydroxyvalerate (Tianan ENMAT Y1000P) were purchased from Helian Polymers (Venlo, Netherlands). The molecular weight of the copolymer was $215505 \mathrm{gmol}^{-1}$, with a melting point and equilibrium melting temperature of $173{ }^{\circ} \mathrm{C}$ and $208^{\circ} \mathrm{C}$, respectively $[34,38]$.

\section{Experimental procedure}

This paper applies the data obtained by Kelly et al. [27] to a range of crystallization kinetic models. For convenience, the experimental procedure used to obtain these data is reported briefly here. 
Plaques of PHB-co-HV were generated via compression molding using a Moore E1127 hydraulic hot press (George E. Moore \& Sons Ltd, Birmingham). PHB-co-HV (8g) was placed into a mold $(152 \times 158 \times 0.266 \mathrm{~mm})$ and inserted into the press at $190{ }^{\circ} \mathrm{C}$. The polymer was left to melt for $5 \mathrm{~min}$ before a load of 10 tons was applied for an additional $3 \mathrm{~min}$. The plaques were allowed to cool in the press for $20 \mathrm{~min}$.

A Mettler Toledo DSC1 differential scanning calorimeter (Mettler Toledo, Schwerzenbach, Switzerland) was used to determine the crystallization kinetics of PHB-co-HV across a range of temperatures $\left(138-146^{\circ} \mathrm{C}\right)$ under nitrogen. This temperature range was limited by the rapid crystallization kinetics at lower temperatures and the propensity for degradation during prolonged periods at higher temperatures [34]. Discs $(\varnothing 5 \mathrm{~mm})$ were punched out from the plaques, and two were analyzed for each test to give a mass of approximately $10 \mathrm{mg}$. The discs were placed into $40 \mu \mathrm{L}$ aluminum pans and capped with aluminum lids (Mettler Toledo) before being sealed with a press. Samples were held at $210^{\circ} \mathrm{C}$ for $2 \mathrm{~min}$ under nitrogen to melt the polymer before being cooled to the crystallization temperature at a rate of $30{ }^{\circ} \mathrm{Cmin}^{-1}$. The samples were held at this temperature for approximately six half-lives, as estimated from the minima in the crystallization exotherm. This timescale was chosen to obtain enough data from the secondary crystallization-only region while minimizing the degradation of the polymer due to prolonged heating at elevated temperatures. Initial studies indicated the presence of degradation when PHB-co-HV was heated at $146^{\circ} \mathrm{C}$ for in excess of $500 \mathrm{~min}$ (data not shown). Three samples were analyzed for each temperature (labeled A, B, and C). A heat of fusion of $140 \mathrm{Jg}^{-1}$ was used to calculate the fractional crystallinity, as in the absence of a published value for PHB-co-HV, this value has been adopted by many others when analyzing copolymers containing low hydroxyvalerate contents [39-41].

\section{Evaluation of crystallization kinetic models}

The data, following baseline and induction time corrections, obtained by Kelly et al. [27] was used to analyze each of the crystallization kinetic models. For the Avrami, simplified Hillier, Tobin, and Hay models, the parameters were determined via the traditional method of creating double log plots, as discussed in more detail above. In addition, the Makin parameters were calculated from the Avrami results (Eqs. (5) and (6)). These parameters were then applied to the respective models to generate fraction crystallinity versus time plots, and the results were compared to the experimental data using SPSS software (IBM, Portsmouth, UK). The visual fit for the curves was evaluated along with the coefficient of determination $\left(\mathrm{R}^{2}\right)$ values and standard error of regression.

SPSS software was also used to perform direct curve fittings for each of the models using nonlinear regression methods and to calculate the respective kinetic parameters. These parameters were again used to generate cumulative crystallization curves, and the results were evaluated in the same way.

To enable visual comparisons between the models, all cumulative data were converted to the actual fractional crystallinity (Equation (11)) instead of the traditional method of normalizing data to the final crystallinity.

Fractional crystallinity $=\frac{\text { Cumulative area under the exotherm }(\mathrm{Ws} / \mathrm{g})}{\text { Heat of fusion }(\mathrm{J} / \mathrm{g})}$

\section{Results and discussion}

The Avrami, simplified Hillier, Tobin, and Hay parameters were determined through the traditional method of generating double log plots, and SPSS software was used to determine the fit of these parameters to the experimental data. In addition, the Malkin parameters were calculated directly from the Avrami results (Eqs. (5) and (6)).

Figure 1 illustrates the fit of these models to the crystallization kinetics for PHB-co-HV at $138^{\circ} \mathrm{C}$. Similar results were obtained per repeat measurement (A, B, and C) and temperature (data not shown). The results highlight that although the models are able to sufficiently describe the primary crystallization, there is an inability to accurately model the region dominated by the secondary crystallization process $\left(X_{t}>0.6\right.$, as determined by the Hay model). The exception to this is the Hay model, which is designed to include contributions from both primary and secondary crystallization throughout the entire curve.

Additionally, SPSS software was used to directly curve fit the data to the respective models using nonlinear, multivariable regression analysis (Fig. 2). The results show that the Hay and Velisaris-Seferis models are most effective in describing the crystallization kinetics of PHB-co-HV. This is not surprising, as these theories have been developed to consider both primary and secondary crystallization, occurring concurrently or in series, and, therefore, more accurately represent the experimental data. Again, similar observations were found for each temperature analyzed (data not shown). It is also important to note that when curve fitting is used, the accuracy in the modeling of the primary crystallization region is reduced for those models that do not consider the secondary process, most notably the Avrami, simplified Hillier, and Malkin models. This is because the curve fitting process aims to minimize the level of error throughout the entire curve.

A more sophisticated approach to compare these models is to consider the coefficient of determination $\left(\mathrm{R}^{2}\right)$ values (Supplementary information S1-8). Each of the models generated $\mathrm{R}^{2}$ values in excess of 0.9 , showing a good fit to 
Fig. 1 Comparison of the Avrami, Simplified Hillier, Tobin, Malkin and Hay models with experimental data obtained for PHB-co-HV at $138^{\circ} \mathrm{C}$.

Cumulative fractional crystallization curves generated by imputing the calculated crystallization kinetic parameters (Supplementary Information S1-4 and S8) into the respective models. Solid black line corresponds to the experimental data and the model curves are as follows: Avrami dark blue dots; Simplified Hillier - red short dashes; Tobin - green long dashes; Malkin - pink dash and dot; Hay - light blue dash and double dot

Fig. 2 Nonlinear multivariable curve fitting of the kinetics models to the isothermal crystallization of PHB-co-HV at $138^{\circ} \mathrm{C}$. Solid black line corresponds to the experimental data and the model curves are as follows: Avrami - dark blue dots; Simplified Hillier - red short dashes; Tobin - green medium dashes; Malkin - pink short dash and dot; UrbanoviciSegal - light green long dashes; Velisaris-Seferis Parallel orange long dash and dot; Velisaris-Seferis Series - dark orange long dash and dot; Hay light blue dash and double dot
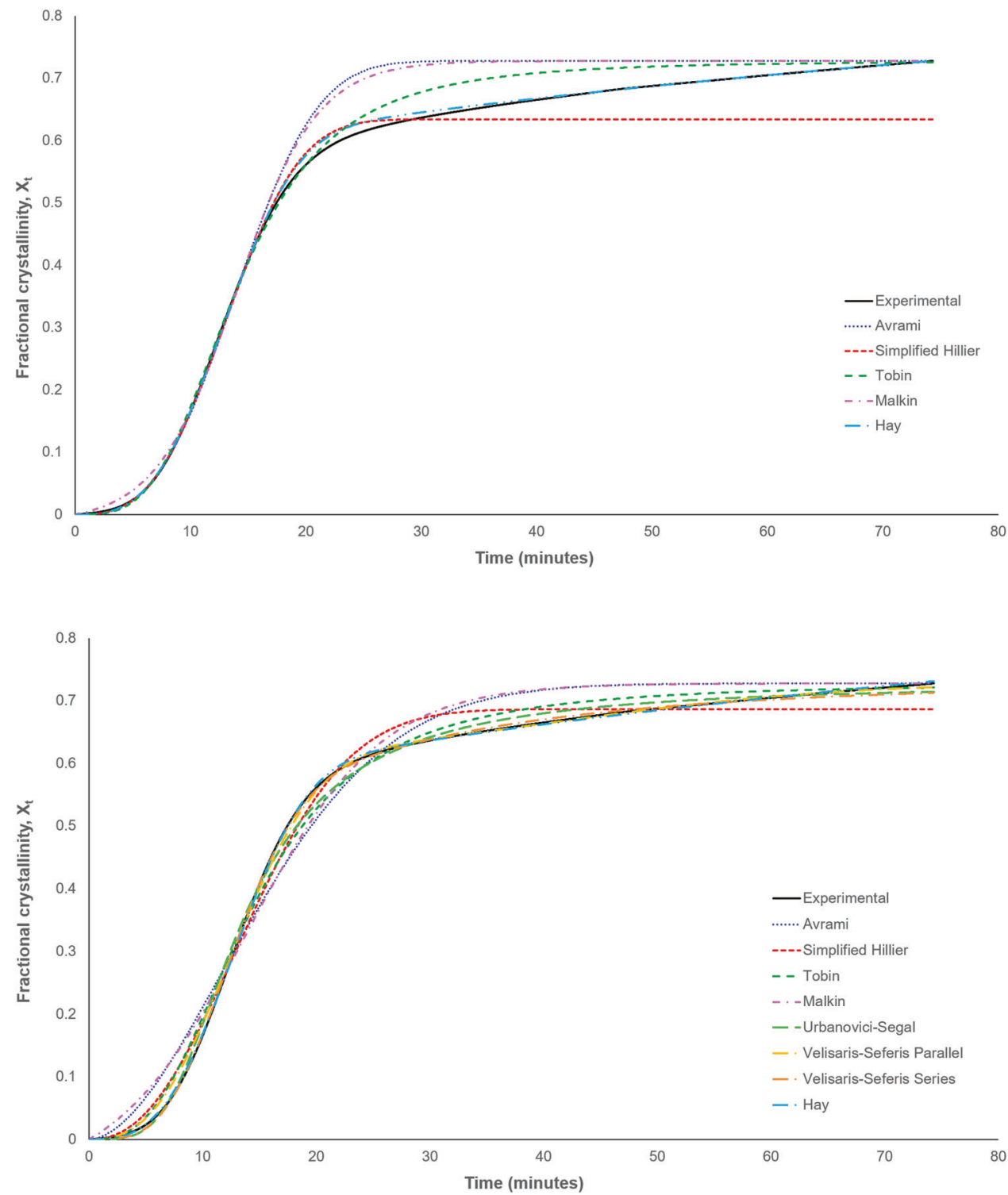

the experimental data. In addition, the results obtained from direct curve fitting gave higher $\mathrm{R}^{2}$ values due to the whole curve being considered; however, this also leads to a deterioration in the quality of the fit for the primary crystallization region (Figs. 1 and 2). This is especially prevalent for the Avrami and Malkin models, which are based on primary crystallization alone. The Urbanovici-Segal, Velisaris-Seferis, and Hay models all generated high $\mathrm{R}^{2}$ values in excess of 0.997 , with the Hay model proving the most effective returning $\mathrm{R}^{2}$ values of 1 for every sample when applying the curve fitting procedure.

As shown in Fig. 2, a high $\mathrm{R}^{2}$ value does not necessarily result in a more accurate representation of the experimental data, especially for the case of models based on primary crystallization alone. It is, therefore, important to also consider individual regions of the traces. Four areas of interest from the cumulative curve were analyzed using the parameters obtained from the double log plot and curve fitting procedure, and the standard error of the regression, $s$, was calculated (Eq. (12)). These regions correspond to the initial stages of crystallization, the primary dominant region, the transition from primary to secondary crystallization, and the secondary crystallization region.

$$
s=\sqrt{ } \frac{\sum\left(X_{t}-X_{t}^{\prime}\right)^{2}}{n-2}
$$

where $s$ is the standard error of the regression, $X_{t}$ is the experimental fractional crystallinity, $X_{t}^{\prime}$ is the modeled fractional crystallinity and $n$ is the number of data points.

For the purpose of comparing these models, the region corresponding to the initial stages of crystallinity was defined to be below $10 \%$ crystallinity $\left(0<X_{t}<0.1\right)$. The parameters derived from the double log plots generated a 
Table 1 Average standard error of regression for each of the models over selected regions of the crystallization curve

\begin{tabular}{|c|c|c|c|c|c|c|c|c|c|c|c|c|c|}
\hline \multirow[t]{3}{*}{ Region } & \multirow{2}{*}{\multicolumn{2}{|c|}{ Avrami }} & \multirow{2}{*}{\multicolumn{2}{|c|}{$\begin{array}{l}\text { Simplified } \\
\text { Hillier }\end{array}$}} & \multirow{2}{*}{\multicolumn{2}{|c|}{ Tobin }} & \multirow{2}{*}{\multicolumn{2}{|c|}{ Malkin }} & \multirow{3}{*}{$\begin{array}{l}\text { Urbanovici-Segal } \\
\mathrm{CF}\end{array}$} & \multicolumn{2}{|c|}{ Velisaris-Seferis } & \multirow{2}{*}{\multicolumn{2}{|c|}{ Hay }} \\
\hline & & & & & & & & & & Parallel & Series & & \\
\hline & DLP & $\mathrm{CF}$ & DLP & $\mathrm{CF}$ & DLP & $\mathrm{CF}$ & DLP & CF & & $\mathrm{CF}$ & $\mathrm{CF}$ & DLP & $\mathrm{CF}$ \\
\hline Initial & 0.001 & 0.027 & 0.002 & 0.014 & 0.003 & 0.008 & 0.012 & 0.033 & 0.007 & 0.024 & 0.016 & 0.002 & 0.004 \\
\hline Primary & 0.007 & 0.018 & 0.004 & 0.013 & 0.006 & 0.010 & 0.007 & 0.021 & 0.012 & 0.013 & 0.012 & 0.004 & 0.004 \\
\hline Transition & 0.069 & 0.034 & 0.016 & 0.024 & 0.022 & 0.017 & 0.060 & 0.033 & 0.017 & 0.012 & 0.017 & 0.014 & 0.004 \\
\hline Secondary & 0.028 & 0.027 & 0.051 & 0.018 & 0.020 & 0.015 & 0.028 & 0.027 & 0.011 & 0.005 & 0.012 & 0.002 & 0.002 \\
\hline
\end{tabular}

$D L P$ curve produced from parameters calculated through double log plots, $C F$ Curve produced via curve fitting by SPSS

Fig. 3 Primary and secondary crystallization plots extracted from the cumulative curve.

"Total" denotes the experimental data, and "Primary" and "Secondary" denote the calculated curves

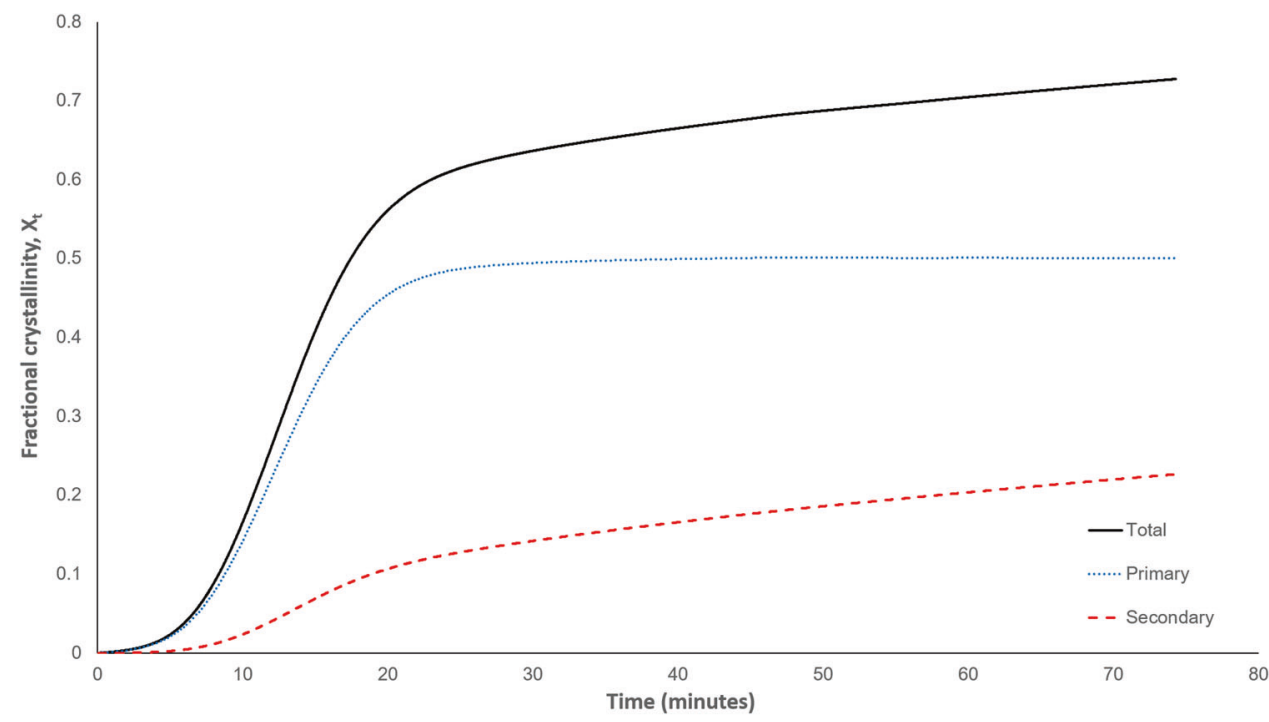

lower standard error for the regression than direct curve fitting for the majority of the models evaluated (Table 1, Supplementary information S9). When applying the curve fitting procedure, SPSS software takes into consideration the entire plot, and is, therefore, unable to effectively process those models that focus mainly on primary crystallization. This is evident when comparing the difference in the errors between the curves calculated from the double log parameters and those from the curve fitting procedure for the Avrami and Hay models. The Avrami, simplified Hillier and Hay models, using parameters calculated from the double log plots, were found to provide the closest fit to the experimental data.

The region where primary crystallization dominates $\left(0.25<X_{t}<0.45\right)$ is taken around the inflection point of the crystallization curve, as this is where the rate of primary crystallization is greatest. In this region, the curves derived from the double log plots again proved to be the most effective (Table 1, Supplementary information S10). The simplified Hillier, using the calculated parameters, and both versions of the Hay method were found to be most suitable for modeling the primary crystallization with the standard error for the regression generally calculated to be less than 0.005 . Both of these methods dictate the completion of primary crystallization and analyze this portion independently, which is perhaps why these models are the most successful.

The majority of the models were unable to successfully describe the transition from primary to secondary crystallization, with greater standard errors observed compared to the other regions (Table 1, Supplementary information S11). In general, results obtained using parameters calculated from the double log plots gave greater standard errors of regression than curve fitting due to the transitional region being rarely considered within models. As the Tobin, Urbanovici-Segal, Velisaris-Seferis, and Hay models account for the secondary crystallization process, they were more successful than those solely based on primary crystallization, with the SPSS curve fitting of the Hay model proving to be significantly superior.

Only the parallel form of the Velisaris-Seferis and the Hay equations could be used to successfully model the secondary crystallization portion of the curves (Table 1, Supplementary information S12) with standard errors of regression of 0.005 or less obtained. This is to be expected, as these are the only models that account for the presence of both primary and secondary crystallization.

Interestingly, the parallel form of the Velisaris-Seferis model outperformed the series method over the majority of 
the selected areas. This confirms the Hay theory that both primary and secondary crystallization occur concurrently [27] and provides an explanation as to why the Hay model significantly improves on the other models when studying across the entire crystallization curve. Manipulation of the Hay equation can also enable the primary and secondary aspects of the cumulative crystallization curve to be plotted in isolation, highlighting the parallel nature of the two processes (Fig. 3).

\section{Assessment of the parameters}

One assumption in the Avrami equation is that the $\mathrm{n}$ value should be an integer that directly relates to the nucleation and growth mechanisms occurring during primary crystallization. It has been well documented that integer values are rarely observed [8, 14], and this has been found in this work with both the Avrami and simplified Hillier approaches (Supplementary information S1 and S2). When curve fitting was used to calculate the Avrami exponent, the values deviated even further from an integer number as a result of the inability to consider the secondary crystallization region. The Hay model generated an integer value of 3.0 for all of the samples analyzed, but again, the $\mathrm{n}$ value was reduced by up to 0.2 when direct curve fitting was used (Supplementary information S8). Unlike the Avrami exponent, the Tobin $\left(\mathrm{n}_{\mathrm{t}}\right)$ (Supplementary information S3) and Urbanovici-Segal $\left(\mathrm{n}_{\mathrm{us}}\right)$ (Supplementary Information S5) exponents do not need to be integer values. The Malkin parameter $\mathrm{C}_{0}$ can be calculated from the Avrami exponent, and, therefore, should also be an integer; in this case, 60. Although the results are more precise, an integer value was not obtained (Supplementary information S4). In addition, the curve fitting procedure for the Malkin model gives considerably lower $\mathrm{C}_{0}$ values, proving that this method is unsuccessful (Supplementary information S4). As the Velisaris-Seferis method is based on the Avrami equations, $\mathrm{n}_{\mathrm{vs}}$ should also be an integer, which is again not observed from the curve fitting procedure (Supplementary information S6 and S7). In addition, a wide variation in the exponential values is found between the repeat measurements and different temperatures, highlighting that high $\mathrm{R}^{2}$ values in the curve fitting procedure do not guarantee the calculation of the correct values for the parameters.

\section{Analysis of the primary region alone}

Evaluation of the models thus far has focused on a polymer that shows significant secondary crystallization. As most of the models assessed have been developed for primary crystallization, it is not surprising that they do not adequately describe the entire crystallization process effectively. The same crystallization-time curves were all reassessed using those models that emphasize primary crystallization (Avrami, simplified Hillier, Tobin, Malkin, and Urbanovici-Segal), with the data limited to this region alone. Modeling of the Avrami equation for a nominal range of time points shows that for an ' $n$ ' value of three, the rate of primary crystallization minimizes at a time equivalent to double the half-life of crystallization [27]. If the ' $n$ ' value is two, this increases to triple the half-life. Therefore, if the data are restricted to a time period of triple the half-life (as estimated from the minima of the exotherm), one can be confident that it mainly contains primary crystallization.

The majority of the results from this analysis are presented in the supplementary information (S13-20). As shown by the cumulative curves created by the models, this restriction of the data improves the effectiveness of the models (Fig. 4).

This is reflected in an increase in the $\mathrm{R}^{2}$ values for each of the models (Supplementary information S13-16), with the exception of the Urbanovici-Segal model (Supplementary information S17). In addition, the curve fitting procedure produced $\mathrm{R}^{2}$ values ranging between 0.999 and 1 for the simplified Hillier and Tobin models across the majority of samples analyzed. In most cases, the Avrami exponent was also found to be approximately equal to an integer value of three. Despite the improvement in the models when only considering the primary portion of the curve, the Hay model still gave the highest $\mathrm{R}^{2}$ values using both calculated parameters and curve fitting. This again highlights the concurrent nature of primary and secondary crystallization and the requirement to analyze both processes in isolation.

Comparison of the standard error of regression for defined areas of the crystallization curve also pointed to an improvement when only the primary region was considered (Supplementary information S18-20). A marginal improvement in the region corresponding to the initial stages of crystallization was found for those curves produced from the parameters calculated by the double log plot; however, a large reduction in the standard error of the regression was found from the curve fitting procedure. This is because the fitting procedure is no longer distorted by a large degree of secondary crystallization that is not accounted for by the models. The greatest improvement was found where the rate of primary crystallization is at a maximum. With the exception of the Urbanovici-Segal model, large reductions to the standard error of regression were observed from both the calculated and curve fitting procedures compared to the full crystallization curve. The improvement in the transition region is inconsistent across each of these models, as this process is not considered by those models focusing mainly on primary crystallization.

Restricting the data to consider only the region where the primary mechanism dominates improves the ability of Avrami, simplified Hillier, Tobin, and Malkin models to model the crystallization kinetics of PHB-co-HV with high $\mathrm{R}^{2}$ values, and, where applicable, $n_{\mathrm{a}}$ values close to three are 


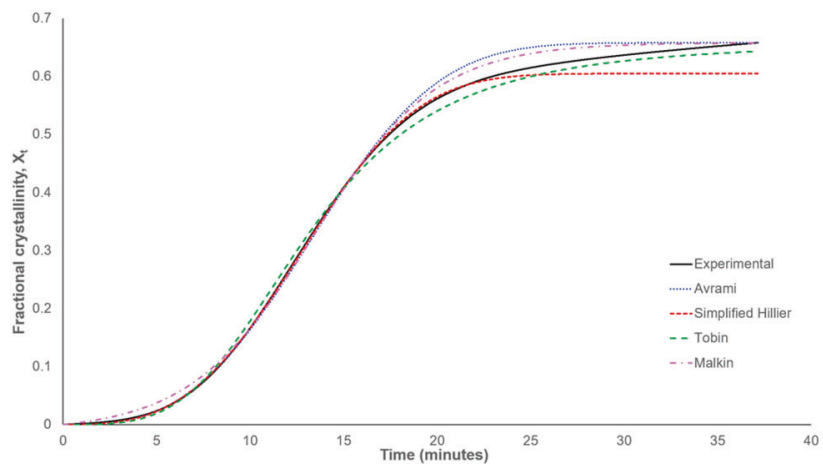

(a)

Fig. 4 Comparison of the Avrami, Simplified Hillier, Tobin, Malkin, and Urbanovici-Segal models with experimental data in the primary crystallization region: a Curves generated by imputing the crystallization kinetic parameters calculated from double log plots (b) Nonlinear multivariable curve fitting of the kinetic models. Solid black line

obtained (Supplementary information S13-16). However, as the above results show, the models are still unable to account for the entire curve; in particular, the transition from primary to secondary crystallization. Comparison across all the studies performed shows that the Hay model is the most successful at describing the crystallization kinetics of PHB-co-HV and most likely any polymer containing a high degree of secondary crystallization.

\section{Conclusions}

This work highlights the importance of selecting the optimum model when analyzing the crystallization kinetics of polymers, especially those displaying high levels of secondary crystallization.

The isothermal crystallization of PHB-co-HV was evaluated using a range of models, namely, Avrami, simplified Hillier, Tobin, Malkin, Urbanovici-Segal, Velisaris-Seferis, and Hay. Two methods of model evaluation were used: determination of the parameters through traditional double log plots and nonlinear, multivariable regression to perform curve fittings. The results show that although the curve fitting method produces higher $\mathrm{R}^{2}$ values, modeling of the primary region is less accurate than when the parameters are calculated from double log plots. The exception to this is the Hay model, which is designed to account for both primary and secondary crystallization occurring simultaneously.

The Hay and Velisaris-Seferis models were found to be the most successful at modeling the entire crystallization curve as a result of incorporating the secondary crystallization process. In addition, the parallel form of the Velisaris-Seferis model outperforms the series model, confirming the Hay theory that primary and secondary crystallization occur concurrently. When assessing the parameters returned by the models, the Avrami exponent

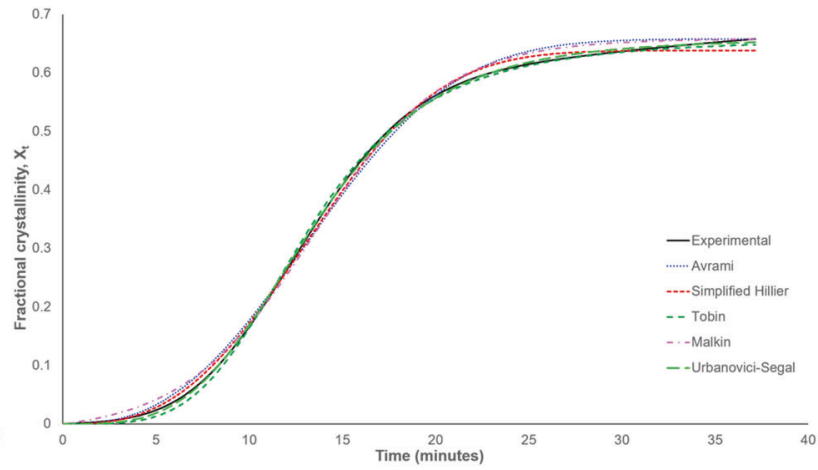

(b)

corresponds to the experimental data and the model curves are as follows: Avrami - dark blue dots; Simplified Hillier - red short dashes; Tobin - green medium dashes; Malkin - pink short dash and dot; Urbanovici-Segal - light green long dashes

was found to have a noninteger value for the Velisaris-Seferis equations and varied vastly between the samples. The Avrami exponent was calculated via a double log plot of the Hay model to be 3. This work shows that the use of the Hay model, and in particular calculation of model parameters through the double log plot, to be the optimum method for analyzing the crystallization kinetics of polymers displaying significant secondary crystallization.

\section{Compliance with ethical standards}

Conflict of interest The authors declare no competing interests.

Publisher's note Springer Nature remains neutral with regard to jurisdictional claims in published maps and institutional affiliations.

Open Access This article is licensed under a Creative Commons Attribution 4.0 International License, which permits use, sharing, adaptation, distribution and reproduction in any medium or format, as long as you give appropriate credit to the original author(s) and the source, provide a link to the Creative Commons license, and indicate if changes were made. The images or other third party material in this article are included in the article's Creative Commons license, unless indicated otherwise in a credit line to the material. If material is not included in the article's Creative Commons license and your intended use is not permitted by statutory regulation or exceeds the permitted use, you will need to obtain permission directly from the copyright holder. To view a copy of this license, visit http://creativecommons. org/licenses/by/4.0/.

\section{References}

1. Rybnikar F. Mechanism of secondary crystallization in polymers. J of Polymer Sci Part a-General Papers. 1963;1:2031-8. https:// doi.org/10.1002/pol.1963.100010620.

2. Chen ZY, Jenkins MJ, Hay JN. Annealing of poly (ethylene terephthalate). Eur Polym J 2014;50:235-42. https://doi.org/10. 1016/j.eurpolymj.2013.11.004.

3. Avrami M. Kinetics of phase change I - General theory. J Chem Phys. 1939;7:1103-12. https://doi.org/10.1063/1.1750380. 
4. Avrami M. Kinetics of phase change. II transformation-time relations for random distribution of nuclei. J Chem Phys. 1940;8:212-24. https://doi.org/10.1063/1.1750631.

5. Avrami M. Granulation, phase change, and microstructure kinetics of phase change. III. J Chem Phys. 1941;9:177-84. https://doi.org/10.1063/1.1750872.

6. Hay JN. Secondary crystallization kinetics. Polym Crystallisation 2018;1:e10007. https://doi.org/10.1002/pcr2.10007.

7. Banks W, Sharples A. The AVRAMI equation in polymer crystallization. Die Makromol Chem. 1963;59:233-6. https://doi.org/ 10.1002/macp.1963.020590119.

8. Supaphol P, Spruiell JE. Application of the Avrami, Tobin, Malkin, and simultaneous Avrami Macrokinetic models to isothermal crystallisation of syndiotactic polypropylene. J Macromol Sci - Phys. 2000;B39:257-77.

9. Hillier IH. Modified Avrami equation for bulk crystallisation kinetics of spherulitic polymers. J Polym Sci Part a-Gen Pap. 1965;3:3067-8. https://doi.org/10.1002/pol.1965.100030902.

10. Ravindranath K, Jog JP. Polymer Crystallization kinetics - Poly (ethylene-terephthalate) and poly(phenylene sulfide). J Appl Polym Sci 1993;49:1395-403. https://doi.org/10.1002/app.1993.070490807.

11. Tobin MC. Theory of phase transition kinetics with growth site impingement. i. homogeneous nucleation. J Polym Sci: Part B: Polym Phys. 1974;12:399-406.

12. Tobin MC. The theory of phase transition kinetics with growth site impingement. II. heterogeneous nucleation. J Polym Sci: Part B: Polym Phys. 1976;14:2253-7.

13. Tobin MC. Theory of phase-transition kinetics with growth site impingement. 3. Mixed heterogenous-homogeneous nucleation and nonintegral exponets of time. J Polym Sci Pt B-Polym Phys. 1977;15:2269-70. https://doi.org/10.1002/pol.1977.180151217.

14. Malkin AY, Beghishev VP, Keapin IA, Bolgov SA. General treatment of polymer crystallisation kinetics-part 1 . a new macrokinetic equation and its experimental verification. Polym Eng Sci. 1984;24:1396-401.

15. Urbanovici E, Segal E. New formal relationships to describe the kinetics of crystallisation. Thermochim Acta. 1990;171:87-94.

16. Urbanovici $\mathrm{E}$, Schneider HA, Brizzolara D, Cantow HJ. Isothermal melt crystallization kinetics of poly(L-lactic acid), J Therm Anal. 1996;47:931-9. https://doi.org/10.1007/bf01979440.

17. Supaphol P. Application of the Avrami, Tobin, Malkin, and Urbanovici-Segal macrokinetic models to isothermal crystallisation of syndiotactic polypropylene. Thermochim Acta 2001;370:37-48.

18. Cruzpinto JJC, Martins JA, Oliveira MJ. The isothermal crystallization of engineering polymers - POM and PEEK. Colloid Polym Sci 1994;272:1-16. https://doi.org/10.1007/bf00653305.

19. Dangseeyun N, Srimoaon P, Supaphol P, Nithitanakul M. Isothermal melt-crystallization and melting behavior for three linear aromatic polyesters. Thermochim Acta 2004;409:63-77. https:// doi.org/10.1016/s0040-6031(03)00331-9.

20. Thanomkiat P, Phillips RA, Supaphol P. Influence of molecular characteristics on overall isothermal melt-crystallisation behaviour and equilibrium melting temperature of syndiotactic polpropylene. Eur Polym J. 2004;40:1671-82.

21. Al-Mulla A. Isothermal crystallization kinetics of poly(ethylene terephthalate) and poly(methyl methacrylate) blends. Express Polym Lett 2007;1:334-44. https://doi.org/10.3144/expresspolymlett.2007.48.

22. Asadinezhad A, Jafari SH, Khonakdar HA, Bohme F, Hassler R, Haussler L. Kinetics of isothermal crystallization and subsequent melting behavior of PTT/PA12 blend. J Appl Polym Sci. 2007;106:1964-71. https://doi.org/10.1002/app.26808.

23. Al-Mulla A, Al-Omairi L, Mathew J, Bhattacharya S. Isothermal crystallization kinetics of tricomponent blends of polycarbonate, poly(trimethylene terephthalate) and polybutylene terephthalate. Polym Polym Compos 2010;18:245-51.
24. Shi XM, Wang JD, Jiang BB, Yang YR. Influence of nanofiller dimensionality on the crystallization behavior of HDPE/carbon nanocomposites. J Appl Polym Sci. 2013;128:3609-18. https:// doi.org/10.1002/app.38581.

25. Khoshkava V, Ghasemi H, Kamal MR. Effect of cellulose nanocrystals (CNC) on isothermal crystallization kinetics of polypropylene. Thermochim Acta 2015;608:30-9. https://doi.org/ 10.1016/j.tca.2015.04.007.

26. Nikam PN, Deshpande VD. Isothermal crystallization kinetics of PET/alumina nanocomposites using distinct macrokinetic models. J Therm Anal Calorim. 2019;138:1049-67. https://doi.org/10. 1007/s10973-019-08192-x.

27. Kelly CA, Hay JN, Turner RP, Jenkins MJ. The effect of a secondary process on the analysis of isothermal crystallisation kinetics by differential scanning calorimetry. Polymers 2020;12:19.

28. Velisaris CN, Seferis JC. Crystallisation kinetics of polyetherethekertone (PEEK) matrices. Polym Eng Sci 1986;26:1574-81. https://doi.org/10.1002/pen.760262208.

29. Albano C, Papa J, Ichazo M, Gonzalez J, Ustariz C. Application of different macrokinetic models to the isothermal crystallization of PP/talc blends. Compos Struct 2003;62:291-302. https://doi. org/10.1016/j.compstruct.2003.09.028.

30. Doi Y. Microbial polyesters, VCH Publishers, New York, 1990.

31. Kelly CA, Overton TW, Jenkins MJ. Syntheis of biodegradable polyhydroxyalkanoates from soil bacteria, in: SK Nayak, BB Mishra, editors. Frontier in soil and environ mental microbiology, CRC Press, Boca Raton, 2020.

32. Bugnicourt E, Cinelli P, Lazzeri A, Alvarez V. Polyhydroxyalkanoate (PHA): Review of synthesis, characteristics, processing and potential applications in packaging. Express Polym Lett 2014;8:791-808. https://doi.org/10.3144/expresspolymlett.2014.82.

33. Biddlestone F, Harris A, Hay JN, Hammond T. The physical ageing of amorphous poly(hydroxybutyrate). Polym Int. 1996;39:221-29.0959-8103/96/\$09.00

34. Jenkins MJ, Robbins KE, Kelly CA. Secondary crystallisation and degradation in PHB-co-HV: an assessment of long-term stability. Polym J. 2018;50:365-73. https://doi.org/10.1038/s41428-0170012-8.

35. Phillipson K, Jenkins MJ, Hay JN. The kinetics of crystallization of poly(epsilon-caprolactone) measured by FTIR spectroscopy. J Therm Anal Calorim. 2016;123:1491-500. https://doi.org/10. 1007/s10973-015-5047-5.

36. Chen ZY, Hay JN, Jenkins MJ. The effect of secondary crystallization on crystallization kinetics - Polyethylene terephthalate revisited. Eur Polym J 2016;81:216-23. https://doi.org/10.1016/j. eurpolymj.2016.05.028.

37. Phillipson K, Jenkins MJ, Hay JN. The effect of a secondary process on crystallization kinetics - Poly (epsilon-caprolactone) revisited. Eur Polym J 2016;84:708-14. https://doi.org/10.1016/j. eurpolymj.2016.09.037.

38. Robbins KE. Secondary crystallisation and degradation during long term aging of $\mathrm{P}(\mathrm{HB}-\mathrm{co}-\mathrm{HV})$, School of Metallurgy and Materials, University of Birmingham, 2016.

39. Avella M, Calandrelli L, Immirzi B, Malinconico M, Martuscelli E, Pascucci B, et al. Novel synthesis blends between bacterial polyesters and acrylic rubber: a study on enzymatic biodegradation. J Environ Polym Degrad 1995;3:49-60. https://doi.org/10. 1007/bf02067793.

40. Thellen C, Coyne M, Froio D, Auerbach M, Wirsen C, Ratto JA. A processing, characterization and marine biodegradation study of meltextruded polyhydroxyalkanoate (PHA) films. J Polym Environ 2008;16:1-11. https://doi.org/10.1007/s10924-008-0079-6.

41. Rosa DD, Calil MR, Guedes CDF, Rodrigues TC. Biodegradability of thermally aged PHB, PHB-V, and PCL in soil compostage. J Polym Environ 2004;12:239-45. 